\title{
Lysyl oxidase expression is associated with inferior outcome and Extramedullary disease of acute myeloid leukemia
}

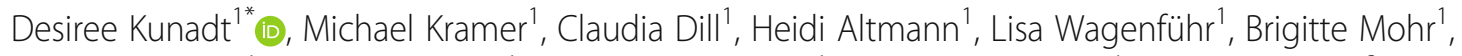
Christian Thiede ${ }^{1}$, Christoph Röllig ${ }^{1}$, Johannes Schetelig ${ }^{1}$, Martin Bornhäuser ${ }^{1}$, Markus Schaich ${ }^{2}$ and Friedrich Stölzel ${ }^{1}$

\begin{abstract}
Background: Lysyl oxidase (LOX) has been described as necessary for premetastatic niche formation in epitheliumderived malignancies and its expression level therefore correlates with risk of metastatic disease and overall survival. However, its role in acute myeloid leukemia (AML) has not been sufficiently analyzed.

Methods: We investigated LOX plasma expression in 683 AML patients (age 17-60 years) treated within the prospective AML2003 trial (NCT00180102). The optimal cut-off LOX value was determined using a minimal-p-value method dichotomizing patients into a LOX-high group (> 109 ng/mL, $n=272,40 \%)$ and a LOX-low group ( $\leq 109$ $\mathrm{ng} / \mathrm{mL}, n=411,60 \%)$.

Results: Higher LOX expression was associated with lower peripheral white blood cells, lower serum LDH, and a lower frequency of FLT3-ITD and NPM1 mutations at diagnosis. Higher LOX expression was found significantly more frequently in patients with secondary AML and therapy-related AML, in patients with French-American-British M5 subtypes, and in patients with adverse-risk cytogenetics. Comparing patients in the LOX-high group and the LOXlow group revealed a 3-year overall survival (OS) of 47 and $53 \%(p=0.022)$ and 3 -year event-free survival (EFS) of 27 and $35 \%(p=0.005)$, respectively. In the LOX-high group significantly more patients had extramedullary AML compared to the LOX-low group $(p=0.037)$. Combining extramedullary AML and LOX as interacting factors in a multivariate analysis resulted in an independent impact on survival for the LOX-high-extramedullary interaction for $\mathrm{OS}(\mathrm{HR}=2.25, p=0.025)$ and $\mathrm{EFS}(\mathrm{HR}=2.48, p=0.008)$. Furthermore, in patients with extramedullary disease $(n=59)$ the LOX level predicted survival. Patients within the LOX-low group had an OS of $43 \%$ and EFS of $36 \%$ as compared to the LOX-high group with an OS of $13 \%$ and EFS of $6 \%$ ( $p=0.002$ and $p=0.008$, respectively).
\end{abstract}

Conclusion: We hypothesize LOX expression to be a new potential biomarker to predict outcome in AML, specifically in AML subgroups such as the prognostic heterogeneous group of AML patients with extramedullary disease.

Trial registration: This retrospective study was performed with patient samples registered within the prospective AML2003 trial (NCT00180102). Patients were enrolled between December 2003 and November 2009.

Keywords: Acute myeloid leukemia, Lysyl oxidase, Extramedullary AML, Survival, Prognosis

\footnotetext{
* Correspondence: desiree.kunadt@uniklinikum-dresden.de

'Department of Internal Medicine I, University Hospital Carl Gustav Carus,

Technical University of Dresden, Fetscherstrasse 74, 01307 Dresden, Germany

Full list of author information is available at the end of the article
}

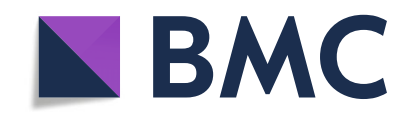

(c) The Author(s). 2020 Open Access This article is licensed under a Creative Commons Attribution 4.0 International License, which permits use, sharing, adaptation, distribution and reproduction in any medium or format, as long as you give appropriate credit to the original author(s) and the source, provide a link to the Creative Commons licence, and indicate if changes were made. The images or other third party material in this article are included in the article's Creative Commons licence, unless indicated otherwise in a credit line to the material. If material is not included in the article's Creative Commons licence and your intended use is not permitted by statutory regulation or exceeds the permitted use, you will need to obtain permission directly from the copyright holder. To view a copy of this licence, visit http://creativecommons.org/licenses/by/4.0/. The Creative Commons Public Domain Dedication waiver (http://creativecommons.org/publicdomain/zero/1.0/) applies to the data made available in this article, unless otherwise stated in a credit line to the data. 


\section{Introduction}

Within the last years various studies were able to demonstrate that in epithelium-derived cancers the occurrence of metastases is an orchestrated process involving hypoxia-induced mechanisms, neo-angiogenesis, recruitment of bone marrow-derived cells, and enzymatic deposition of extracellular matrices which result from certain genetic and epigenetic features of the underlying tumor cells themselves [1-4]. The consecutive formation of a so called premetastatic niche has been subject of studies which showed that abrogation of this premetastatic niche is able to eliminate the metastatic process itself in animal models [5].

Interestingly, these mechanisms have only been described for epithelial tumors so far. However, there is an increasing body of evidence that extramedullary manifestation of acute myeloid leukemia (AML) is often underreported at initial diagnosis and during relapse [6]. Studies have reported differences in frequency of clinically apparent extramedullary manifestations in adult AML between the time of diagnosis (approximately 9\%) compared to relapse after allogeneic hematopoietic stem cell transplantation (HSCT) (5-12\%) and relapsed AML after donor lymphocyte infusion or haploidentical HSCT (up to 32\%) [7-12]. In a prospective trial using total body 18FDG-PET/CT imaging we could determine a $19 \%$ prevalence of metabolically active extramedullary AML at diagnosis with $60 \%$ of histologically confirmed extramedullary sites still being positive in follow-up imaging after intensive AML therapy [13].

While it is speculated that the true prevalence has yet to be defined, some reports have associated extramedullary AML with certain clinical and morphological features such as high white blood cell (WBC) count and French-American-British (FAB) M4/M5 subtypes [1416] as well as distinct cytogenetic features such as $11 \mathrm{q} 23$ abnormalities, monosomy of chromosome 7, and abnormalities of chromosome 8 (most often trisomy 8) [1519]. Published data show a high prevalence of extramedullary AML in nucleophosmin 1 (NPM1) mutated AML patients as well as mutated NPM1 in biopsied extramedullary sites in a high fraction of patients with extramedullary AML [20, 21]. In the subset of normal karyotype AML with NPM1 mutations Garzon et al. were able to demonstrate that a significant proportion of patients have an up-regulation of microRNA 10a (miR-10a) in their leukemic cells [22]. While we were able to confirm these data, we demonstrated further that a significant proportion of these patients have extramedullary involvement at the time of diagnosis. MiRNA microarray data of these AML patients suggested an expression pattern of miRNAs that was also described in regulation processes for the initiation and the maintenance of a pre-metastatic niche. Furthermore, miR10a has a putative binding site to the 3'UTR of lysyl oxidase (LOX) which in return led to this work [23].

LOX is a copper-dependent, extracellularly secreted amine-oxidase mapped on human chromosome 5q23 that has been extensively described to be involved in the initiation of a premetastatic niche in epithelium-derived malignancies and in bone marrow fibrosis as well as in the development of pediatric acute megakaryoblastic leukemia [5, 24, 25]. Dawes et al. were able to increase LOX expression in the Molm14 AML cell line due to hypoxia exposure [26]. LOX expression is increased in tumor cells through hypoxia-inducible factor-1 (HIF-1) stimulation [27, 28]. After secretion of the LOX proenzyme into the extracellular space, proteolytic cleavage which is mainly catalyzed by bone morphogenic protein1 (BMP-1) results in an active $30 \mathrm{kDa}$ enzyme. LOX is thought to mainly catalyze deamination of peptidyl lysine residues in collagen and elastin molecules. Next, these resulting aldehydes undergo spontaneous reactions with other LOX-derived aldehydes or lysine residues. This results in cross-linking of collagen and elastin, which is essential for the stability of collagen fibers and integrity and elasticity of elastin fibers. It has been shown that LOX-dependent crosslinking of collagen fibers is involved in creating a "growth-permissive fibrotic microenvironment" for metastatic growth and the opportunity of tumor cell persistence [29]. Therefore, LOX expression levels correlate with distant metastasis-free and overall survival (OS) in various epithelium-derived cancers [5, 30]. Furthermore, animal models have demonstrated that different approaches inhibiting LOX have been able to sufficiently eliminate metastasis [5].

To date, little is known about the clinical implication of LOX expression in AML. In this study we investigated the plasma LOX expression from AML patients included in the AML2003 trial at diagnosis and correlated the results with clinical features and outcome of these patients.

\section{Material and methods}

All available peripheral blood lithium-heparin plasma samples of 683 patients with AML (age 17-60 years) were analyzed for LOX expression. Lithium-heparin plasma samples were collected at initial diagnosis whenever possible. All patients were treated within the prospective, randomized, and multicentric AML2003 trial (NCT00180102). The study had been approved by the institutional review board (IRB) of the University of Dresden and all IRBs of participating centers of the Study Alliance Leukemia (SAL). The protocol was in agreement with the Helsinki Declaration. Written informed consent was obtained from each patient before inclusion. The risk-adapted design and inclusion criteria of the AML2003 trial have been described previously 
[31]. Briefly, patients were randomly assigned to receive either three cycles of high-dose cytarabine (HD-AraC) or MAC/MAMAC/MAC (mitoxantrone, amsacrine and high-dose cytarabine) as chemo-consolidation in a first step and in a second step to receive either an intensified, risk adapted and priority based consolidation strategy including early allogeneic hematopoietic stem cell transplantation (HSCT) within induction therapy-induced aplasia and autologous HSCT as second consolidation therapy or a standard consolidation strategy with allogeneic HSCT as first consolidation therapy resulting in four treatment arms. In total, 1179 patients between 16 and 60 years with newly diagnosed AML (excluding acute promyelocytic AML) or with myelodysplastic syndrome with excess blasts >9\% (MDS-EB2) were randomized at diagnosis and included in the AML2003 trial. Eligibility was based on confirmation of AML or MDS-EB2 by central morphologic and immunophenotyping analysis.

Bone marrow samples were processed in reference laboratories of the SAL study group. Cytogenetic analyses were performed using standard G-banding techniques and karyotyping according to the International System for Human Cytogenetic Nomenclature [32]. Fluorescent in situ hybridization (FISH) techniques were performed according to the manufacturer's recommendations. Molecular analyses for mutations of FLT3-ITD and NPM1 mutations were performed as previously published in detail [33, 34]. Extramedullary AML was defined according to the World Health Organization (WHO) 2008 criteria requiring a tissue sample from an extramedullary site (i.e. anatomical site other than the bone marrow) composed of myeloid blasts destroying the tissue architecture [35]. Data was collected and certified by the SAL Data Center.

Peripheral blood lithium-heparin plasma samples were analyzed for LOX concentration using an enzymatic assay (Amplite Fluorimetric LOX Assay Kit. AAT Bioquest, Sunnyvale, CA, USA) according to the manufacturer's instructions [36]. All fluorescence reads were performed in triplicate with recombinant human LOXL2 (R\&D Systems, Minneapolis, MN, USA) for standard curve estimation. Signals were read by a fluorescence microplate reader at Ex/Em 540/590 nm (Berthold Technologies, Bald Wildbad, Germany). Supernatant of the NPM1 mutated AML cell line OCI/AML3 served as internal control and for analysis of a day-to-day variability. OCI/AML3 cells were purchased from the LeibnitzInstitute - German Collection of Microorganisms and Cell Cultures (DSMZ, Braunschweig, Germany).

\section{Statistical analysis}

Overall survival (OS) and event-free survival (EFS) were measured from the date of entry into the study to the date of event or the last follow up. Death, induction failure, and relapse were considered events for EFS. Complete remission (CR) was defined according to standard criteria [37]. The range of LOX serum concentration for all patients was $0-2184 \mathrm{ng} / \mathrm{mL}$ with a mean LOX concentration of $119.7 \mathrm{ng} / \mathrm{mL}$. Inspection of the Martingale residuals of a Cox model testing the influence of LOX as a continuous variable on OS revealed that a cut-off model might be most appropriate. The optimal cut-off LOX value was determined using a minimal- $p$-value method [38]. The resulting logarithmic $\operatorname{logLOX}$ value $=2.0403 \quad(109 \mathrm{ng} / \mathrm{mL})$ was used to dichotomize all patients into a LOX-high group (>109 $\mathrm{ng} / \mathrm{mL}, n=272,40 \%)$ and a LOX-low group ( $\leq 109 \mathrm{ng} /$ $\mathrm{mL}, n=411,60 \%)$. Differences of continuous variables between both groups were analysed by means of the Mann-Whitney-U-Test. Differences of the distribution of categorical variables were analysed using the uncorrected Chi-Squared-Test. The method of Kaplan-Meier was used to estimate OS and EFS. Survival distributions were compared using the log-rank test. Prognostic parameters were tested in a Cox regression model for OS and EFS. Cytogenetic karyotype, FLT3-ITD mutation status, NPM1 mutation status, extramedullary AML, and LOX expression at diagnosis were used as dichotomized variables whereas age, and logarithmic (to base 10) WBC at diagnosis, and the bone marrow blast count at day 15 after induction chemotherapy were used as continuous variables. All statistical analyses were performed using SPSS version 19.0 (SPSS, Chicago, IL, USA) and R 2.15.1.

\section{Results}

\section{LOX expression and patient characteristics}

For internal validation we first identified the OCI/AML3 cell line as representing a cell line with a high LOX expression in the supernatant. We performed independent supernatant day-to-day variability measurements in triplicate on each day on three consecutive days resulting in a mean LOX concentration of $792.5 \mathrm{ng} / \mathrm{mL}$ and a standard deviation of $101.9 \mathrm{ng} / \mathrm{mL}$ with a day-to-day variability of $13 \%$.

As mentioned, a logarithmic logLOX value was identified which dichotomizes the analyzed 683 patients in a LOX-low group comprised of 411 patients (60\%) and a LOX-high group with 272 patients (40\%). Detailed patient characteristics are depicted in Table 1. Comparing clinical and laboratory baseline characteristics at initial diagnosis AML patients in the LOX-high group exposed significantly less bone marrow blasts $(54 \%$ vs. $63 \%, p<$ $0.001)$, lower WBC counts $(6.5 \mathrm{GPt} / \mathrm{L}$ vs. $26 \mathrm{GPt} / \mathrm{L}, p<$ 0.001 ), and lower serum LDH (389 U/l vs. $5247 \mathrm{U} / \mathrm{l}, \mathrm{p}<$ 0.001 ) compared to those patients with low LOX expression, respectively. Furthermore, a significantly lower 
Table 1 Study patients' characteristics

\begin{tabular}{|c|c|c|c|c|}
\hline & $\begin{array}{l}\text { all AML } \\
n=683\end{array}$ & $\begin{array}{l}\text { LOX-low group } \\
n=411,60 \%\end{array}$ & $\begin{array}{l}\text { LOX-high group } \\
n=272,40 \%\end{array}$ & $p$-value \\
\hline $\begin{array}{l}\text { age at diagnosis } \\
\text { median (range) }\end{array}$ & $46(16-60)$ & $47(16-60)$ & $50(18-60)$ & 0.045 \\
\hline Gender, Female, no. (percent) & $364(53)$ & $226(55)$ & $138(51)$ & 0.276 \\
\hline $\begin{array}{l}\text { Bone marrow blasts at diagnosis } \\
\text { [percent] median (range) }\end{array}$ & $60.5(3-100)$ & $63.25(8-100)$ & $54.25(3-96)$ & $<0.001$ \\
\hline $\begin{array}{l}\text { WBC count at diagnosis } \\
\text { [Gpt/L] median (range) }\end{array}$ & $14.5(0.3-353)$ & $26.1(0.3-353)$ & $6.5(0.6-243.4)$ & $<0.001$ \\
\hline $\begin{array}{l}\text { Platelet count at diagnosis } \\
\text { [Gpt/L] median (range) }\end{array}$ & $52(4-1308)$ & $51(4-1308)$ & $52(4-793)$ & 0.626 \\
\hline $\begin{array}{l}\text { Serum LDH at diagnosis } \\
{[\mathrm{U} / \mathrm{l}] \text { median (range) }}\end{array}$ & $492(100-7369)$ & $527(144-5934)$ & $389(100-7369)$ & $<0.001$ \\
\hline FLT3-ITD mutational status positive, no. (percent) ${ }^{a}$ & $167(25)$ & $122(30)$ & $45(17)$ & $<0.001$ \\
\hline NPM1 mutational status positive, no. (percent) ${ }^{b}$ & $226(33)$ & $155(38)$ & $71(26)$ & 0.002 \\
\hline \multicolumn{5}{|l|}{ Combined NPM1/FLT3 status, no. (percent) } \\
\hline NPM1 wt/FLT3 wt & $377(56)$ & $204(50)$ & $173(65)$ & \multirow[t]{4}{*}{$<0.001$} \\
\hline NPM1 wt/FLT3 mut & $70(10)$ & $46(11)$ & $24(9)$ & \\
\hline NPM1 mut/FLT3 wt & $130(19)$ & $80(20)$ & $50(19)$ & \\
\hline NPM1 mut/FLT3 mut & $96(14)$ & $75(18)$ & $21(8)$ & \\
\hline \multicolumn{5}{|l|}{ Disease status at diagnosis, no. (percent) } \\
\hline De novo AML & $603(88)$ & $378(92)$ & $225(83)$ & \multirow[t]{3}{*}{0.001} \\
\hline $\mathrm{t}-\mathrm{AML}$ & $35(5)$ & $17(4)$ & $18(7)$ & \\
\hline mdsAML & $45(7)$ & $16(4)$ & $29(11)$ & \\
\hline \multicolumn{5}{|l|}{ FAB subtypes at diagnosis, no. (percent) } \\
\hline Mo & $27(4)$ & $11(3)$ & $16(6)$ & \multirow[t]{12}{*}{0.001} \\
\hline M1 & $134(20)$ & $96(23)$ & $38(14)$ & \\
\hline M2 & $204(30)$ & $119(29)$ & $85(31)$ & \\
\hline M4 & $88(13)$ & $62(15)$ & $26(10)$ & \\
\hline M4eo & $36(5)$ & $28(7)$ & $8(3)$ & \\
\hline M5a & $71(10)$ & $35(8)$ & $36(13)$ & \\
\hline M5b & $22(3)$ & $11(3)$ & $11(4)$ & \\
\hline M5 other & $7(1)$ & $4(1)$ & $4(1)$ & \\
\hline M6 & $23(3)$ & $9(2)$ & $14(5)$ & \\
\hline M7 & $6(1)$ & $3(1)$ & $3(1)$ & \\
\hline RAEB & $19(3)$ & $9(2)$ & $10(4)$ & \\
\hline RAEB-T & $33(5)$ & $17(4)$ & $16(6)$ & \\
\hline \multicolumn{5}{|l|}{ Cytogenetic subgroups, no. (percent) } \\
\hline adverse risk & $157(23)$ & $65(16)$ & $92(34)$ & \multirow[t]{3}{*}{$<0.001$} \\
\hline intermediate risk & $444(65)$ & $287(70)$ & $157(58)$ & \\
\hline favorable risk & $82(12)$ & $59(14)$ & $23(8)$ & \\
\hline \multicolumn{5}{|l|}{ Common cytogenetic aberrations, no. (percent) } \\
\hline Complex karyotype & $90(13)$ & $42(10)$ & $48(18)$ & 0.005 \\
\hline Normal karyotype & $347(49)$ & $242(59)$ & $95(35)$ & $<0.001$ \\
\hline $\mathrm{t}(8 ; 21)$ & $39(6)$ & $26(6)$ & $13(5)$ & 0.394 \\
\hline inv16 & $43(6)$ & $33(8)$ & $10(4)$ & 0.022 \\
\hline monosomy 5 & $7(1)$ & $3(1)$ & $4(1)$ & 0.347 \\
\hline
\end{tabular}


Table 1 Study patients' characteristics (Continued)

\begin{tabular}{|c|c|c|c|c|}
\hline & $\begin{array}{l}\text { all AML } \\
n=683\end{array}$ & $\begin{array}{l}\text { LOX-low group } \\
n=411,60 \%\end{array}$ & $\begin{array}{l}\text { LOX-high group } \\
n=272,40 \%\end{array}$ & $p$-value \\
\hline del5q & $48(7)$ & $20(5)$ & $28(10)$ & 0.007 \\
\hline monosomy 7 & $30(4)$ & $14(3)$ & $16(6)$ & 0.122 \\
\hline del7q & $20(3)$ & $9(2)$ & $11(4)$ & 0.159 \\
\hline monosomy other than chromosomes 5 or 7 & $43(6)$ & $17(4)$ & $26(10)$ & 0.004 \\
\hline trisomy 8 & $74(11)$ & $31(7)$ & $43(16)$ & 0.001 \\
\hline $\mathrm{t}(9 ; 11)$ & $17(2)$ & $4(1)$ & $13(5)$ & 0.002 \\
\hline abnl(11q23) & $59(9)$ & $22(5)$ & $37(14)$ & $<0.001$ \\
\hline Extramedullary manifestation of AML, no. (percent) & $59(9)$ & $28(7)$ & $31(11)$ & 0.037 \\
\hline
\end{tabular}

Table 1 The study patients' characteristics at diagnosis are listed

Abbreviations: $A M L$ acute myeloid leukemia, FAB French American British classification of acute leukemia, FLT3-ITD, FMS-like tyrosine kinase 3 internal tandem duplication, $L D H$ lactate dehydrogenase, MDS myelodysplastic syndrome, mut mutated, NPM1 Nucleophosmin 1, RAEB refractory anemia with excess blasts, $t$-AML therapy-related AML, mdsAML AML with preceding MDS, WBC white blood count, wt wildtype

${ }^{\text {a }}$ FLT3 mutation screening was performed in $n=678$ patients at initial diagnosis

${ }^{\mathrm{b}}$ NPM1 mutation screening was performed in $n=674$ patients at initial diagnosis

proportion of FLT3-ITD mutations (17\% vs. 30\%, p < $0.001)$ and NPM1 mutations (26\% vs. $38 \%, p=0.002)$ were detected. The LOX-high group consisted of significantly more patients with therapy-related AML (t-AML) (7\% vs. $4 \%)$ and patients with a preceding MDS (mdsAML) (11\% vs. $4 \%$ ), $p=0.001$, a higher proportion of monocytic morphology, more cytogenetically defined adverse-risk karyotypes (34\% vs. 16\%), and less normal karyotypes (58\% vs. $70 \%)$ and favourable karyotypes ( $9 \%$ vs. $14 \%), p<0.001$. In detail, single cytogenetic abnormalities that were identified more frequently among the LOX-high group were complex karyotype (defined as $\geq 3$ independent karyotypic abnormalities) $(p=0.005)$, $\operatorname{del}(5 \mathrm{q})(p=0.007), \mathrm{t}(9 ; 11)(p=0.002)$, and other abnormalities of chromosome 11q23 (abnl (11q23)) $(p<$ 0.001). Abnormalities belonging to a favorable or undefined risk category that were frequently higher expressed in the LOX-high group were monosomies of chromosomes other than chromosome 5 or $7(p=$ $0.004)$, and trisomy of chromosome $8(p=0.001)$. AML with normal karyotype or inversion of chromosome 16 were less frequently observed in the LOX-high group, $p<0.001$ and $p=0.022$, respectively. As hypothesized initially, we could confirm a significantly higher proportion of patients with histologically confirmed extramedullary AML as defined per WHO criteria in the LOXhigh group, $p=0.037$.

\section{Overall- and event-free survival}

Next we analyzed the impact of the LOX-high and LOX-low status on survival in these AML patients. Comparing LOX-high and LOX-low AML patients revealed a 3 -year OS of $47 \%$ (95\% CI $40-53 \%$ ) and $53 \%$ (95\% CI $48-58 \%, p=0.022$ ), and a 3 -year EFS of $27 \%$ (95\% CI $21-32 \%)$ and 35\% (95\% CI 30-40\%, $p=0.005$ ), respectively (Fig. 1a and b). Since the LOX-high group contained significantly more patients with histologically reported extramedullary AML compared to the LOXlow group, $p=0.037$, we aimed to focus further on this subgroup. In order to analyze the impact of LOX expression on survival outcomes adjusting for potential confounding variables in a multivariate analysis, we performed a Cox regression analysis including the prognostic parameters karyotype, age, FLT3-ITD mutation, NPM1 mutation, logarithmic WBC (to base 10), bone marrow blast count at day 15 after induction chemotherapy, extramedullary disease, and LOX expression. In the presence of these variables LOX expression lost its influence on OS and EFS. However, since extramedullary AML and LOX seem to be related on a biological level, we introduced an interaction term in the Cox model. We identified a robust impact of LOX expression and extramedullary manifestation on OS $(\mathrm{HR}=2.25(95 \% \mathrm{CI}$, 1.11-4.56), $p=0.025)$ and EFS (HR $=2.48$ (95\% CI 1.264.86), $p=0.008$ ) (Table 2 ). As previously shown, different forms of consolidation (either 3 cycles of HD-AraC or MAC/MAMAC/MAC as part of the AML2003 trial) did not lead to significant differences in outcome [31].

\section{Subgroup analysis in extramedullary AML}

Since we initially hypothesized that a higher proportion of patients with extramedullary AML would have elevated high LOX plasma levels at diagnosis, we analyzed the cohort of patients with reported and histologically confirmed extramedullary AML at initial diagnosis concurrently with AML without extramedullary leukemic manifestations. In total 59 AML patients with histologically confirmed extramedullary AML at diagnosis and available peripheral blood samples from initial diagnosis could be analyzed for LOX expression. LOX high ( $n=$ 31 ) and LOX low patients $(n=28)$ were about equally distributed. The clinically and laboratory baseline 
A. Overall Survival of LOX-high and LOX-Iow AML patients.

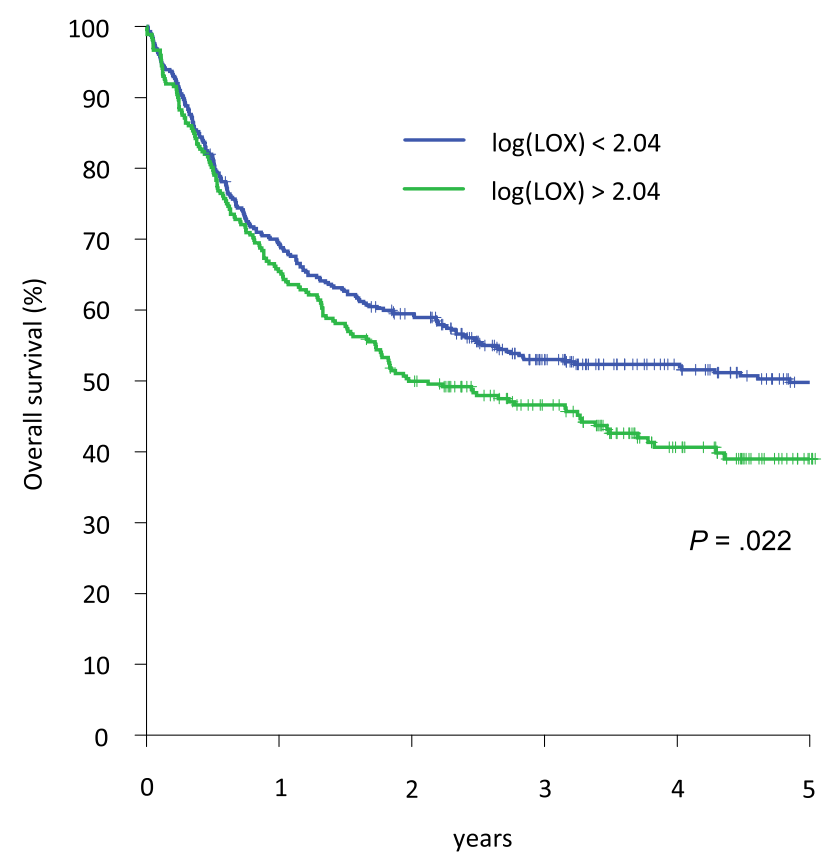

B. Event-free Survival of LOX-high and LOX-low AML patients.

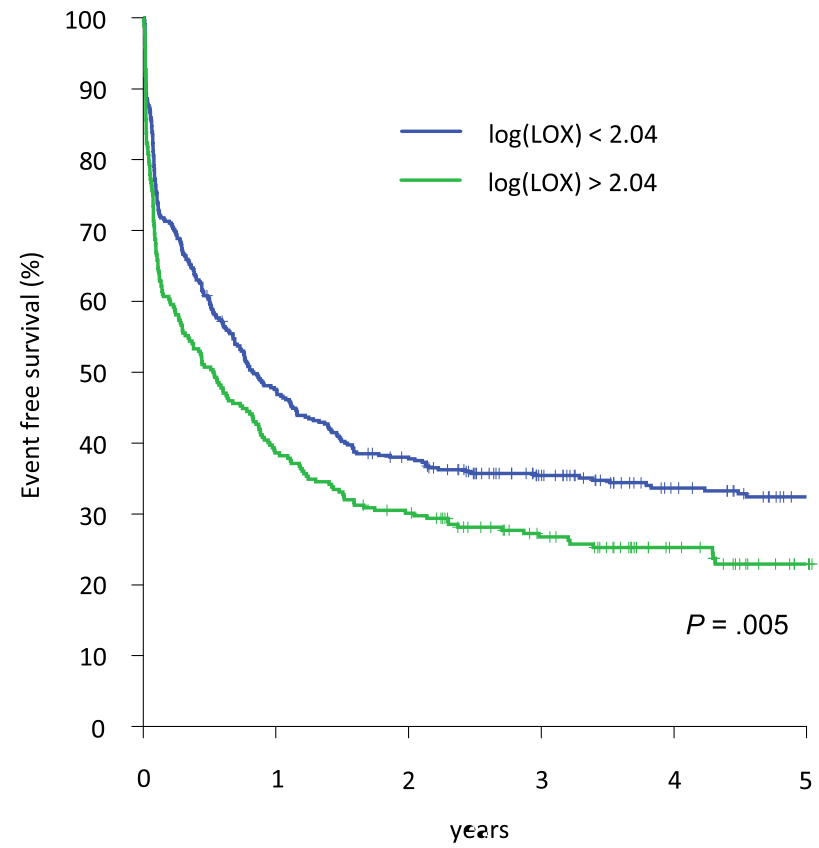

Fig. 1 a and b Overall Survival (OS) (Fig. a) and Event-free Survival (EFS) of 683 AML patients dichotomized to the LOX-high $(n=272)$ and LOXlow $(n=411)$ group. LOX-high patients are denoted in green, LOX-low patients are denoted in blue color. The logarithmic logLOX value $=2.0403$ $(109 \mathrm{ng} / \mathrm{mL})$ was used to dichotomize all patients in both groups, respectively. For LOX-high patients 3-year OS was 47\% (95\% Cl, 40-53\%) and for LOX-low patients $53 \%(95 \% \mathrm{Cl}, 48-58 \%), p=.022$. Three-year EFS for LOX-high patients was 27\% (95\% Cl, 21-32\%) and for LOX-low patients $35 \%(95 \% \mathrm{Cl}, 30-40 \%), p=0.005$ 
Table 2 Multivariable analysis concerning overall and event-free survival

\begin{tabular}{|c|c|c|c|c|}
\hline \multirow[t]{2}{*}{ Factor } & \multicolumn{2}{|c|}{ Overall Survival } & \multicolumn{2}{|c|}{ Event-free Survival } \\
\hline & $\begin{array}{l}\mathrm{HR} \\
{[95 \% \mathrm{Cl}]}\end{array}$ & $\overline{p \text {-value }}$ & $\begin{array}{l}\mathrm{HR} \\
{[95 \% \mathrm{Cl}]}\end{array}$ & $\overline{p \text {-value }}$ \\
\hline Low Risk Karyotype & $\begin{array}{l}0.486 \\
{[0.287-0.825]}\end{array}$ & 0.007 & $\begin{array}{l}0.553 \\
{[0.374-0.818]}\end{array}$ & 0.003 \\
\hline High Risk Karyotype & $\begin{array}{l}1.858 \\
{[1.404-2.459]}\end{array}$ & $<0.001$ & $\begin{array}{l}1.407 \\
{[1.088-1.818]}\end{array}$ & 0.009 \\
\hline Higher Age ${ }^{a}$ & $\begin{array}{l}1.034 \\
{[1.021-1.047]}\end{array}$ & $<0.001$ & $\begin{array}{l}1.021 \\
{[1.011-1.032]}\end{array}$ & $<0.001$ \\
\hline FLT3-ITD positivity & $\begin{array}{l}1.834 \\
{[1.376-2.445]}\end{array}$ & $<0.001$ & $\begin{array}{l}1.430 \\
{[1.109-1.843]}\end{array}$ & 0.006 \\
\hline NPM1 mutation & $\begin{array}{l}0.527 \\
{[0.385-0.723]}\end{array}$ & $<0.001$ & $\begin{array}{l}0.530 \\
{[0.408-0.689]}\end{array}$ & $<0.001$ \\
\hline Logarithmic (to base 10) WBC & $\begin{array}{l}1.191 \\
{[0.962-1.474]}\end{array}$ & 0.109 & $\begin{array}{l}1.030 \\
{[0.857-1.237]}\end{array}$ & 0.754 \\
\hline Bone Marrow Blast Count at Day 15 after Induction Chemotherapy ${ }^{b}$ & $\begin{array}{l}1.010 \\
{[1.005-1.014]}\end{array}$ & $<0.001$ & $\begin{array}{l}1.028 \\
{[1.023-1.033]}\end{array}$ & $<0.001$ \\
\hline Extramedullary Disease & $\begin{array}{l}1.203 \\
{[0.698-2.076]}\end{array}$ & 0.506 & $\begin{array}{l}0.888 \\
{[0.535-1.476]}\end{array}$ & 0.648 \\
\hline high LOX Expression & $\begin{array}{l}0.956 \\
{[0.711-1.285]}\end{array}$ & 0.766 & $\begin{array}{l}1.034 \\
{[0.804-1.329]}\end{array}$ & 0.795 \\
\hline Interaction of Extramedullary Disease and high LOX Expression & $\begin{array}{l}2.246 \\
{[1.106-4.559]}\end{array}$ & 0.025 & $\begin{array}{l}2.478 \\
{[1.264-4.857]}\end{array}$ & 0.008 \\
\hline
\end{tabular}

Table 2 Results of multivariate testing for overall survival and event-free survival including $\mathrm{HR}, 95 \% \mathrm{Cl}$ and $p$-values

Abbreviations: $H R$ hazard ratio, $95 \% \mathrm{Cls} 95 \%$ confidence interval

age as a continuous variable in years

bone marrow blast count as a continuous variable in percent

characteristics of the 59 patients are depicted in Table 3. Interestingly, in this comparison of LOX-high $(n=31)$ and LOX-low $(\mathrm{n}=28)$ patients, only a higher proportion of $\mathrm{t}$-AML and MDS-related AML $(p=0.049)$, higher numbers of monocytic AML according to FAB-subtype $(p=0.003)$, and more patients with cytogenetically defined high-risk abnormalities $(p=0.001)$ remained as statistically significant different in these groups in univariate analysis. Regarding single cytogenetic abnormalities we again observed a two-fold higher rate of complex karyotypes and trisomy 8 in the LOX-high cohort $(n=8,26 \%$ vs. $n=3,11 \%, p=0.137)$. For abnl (11q23) however, a more pronounced significant difference between LOX-high and LOX-low patients was observed despite of low patient numbers in this cohort ( $n=9,29 \%$ vs. $n=1,4 \%, p=0.009)$, respectively. Furthermore, also in patients with extramedullary AML the LOX expression predicted survival. Patients within the LOX-low group had an OS of 43\% (95\% CI, 23-63\%) and $\mathrm{EFS}$ of $36 \%(95 \% \mathrm{CI}, 17-54 \%)$ as compared to the LOX-high group with an OS of $13 \%(95 \% \mathrm{CI}, 1-25 \%)$ and EFS of $6 \%$ (95\% CI, $0-15 \%$ ), $p=0.002$ and $p=0.008$, respectively, in univariate analysis (Fig. 2a and b).

\section{Discussion}

In the current analysis we observed that high LOX expression correlated with the occurrence of extramedullary AML and certain AML features which occur at a high frequency with extramedullary AML. Furthermore, high LOX expression in patients' plasma was found to be associated with statistically significant worse OS and EFS in AML patients under the age of 60 years in a univariate setting - a finding which we found to be even more pronounced in the patient group with reported extramedullary AML. Though, one must speculate that due to the lack of routine screening for extramedullary AML at diagnosis, the prevalence of EM AML might be higher than reported (Stölzel F et al., The prevalence of extramedullary acute myeloid leukemia detected by 18FDG-PET/CT: final results from the Prospective PETAML Trial. Haematologica. 2019 Aug 29. pii: haematol.2019.223032). Consequently, we suggest a considerable number of unknown EM AML patients currently still classified as "normal" AML without clinical apparent extramedullary manifestations at diagnosis for which LOX could also be a potential marker for prognosis.

The multivariate analyses revealed that this association is mainly caused by a higher incidence of adverse cytogenetic aberrations and myelodysplastic features and a lower incidence of favorable cytogenetics and NPM1 mutations.

Furthermore, the statistically significant interaction between higher LOX expression and extramedullary AML 
Table 3 Characteristics of LOX-low/LOX-high extramedullary AML

\begin{tabular}{|c|c|c|c|c|}
\hline & \multicolumn{4}{|l|}{$\begin{array}{l}\text { all EM AML } \\
n=59\end{array}$} \\
\hline & $\begin{array}{l}\text { all EM AML } \\
n=59\end{array}$ & $\begin{array}{l}\text { LOX-low group } \\
n=28,60 \%\end{array}$ & $\begin{array}{l}\text { LOX-high group } \\
n=31,40 \%\end{array}$ & $p$-value \\
\hline $\begin{array}{l}\text { age at diagnosis } \\
\text { median (range) }\end{array}$ & $46(17-60)$ & $45(17-60)$ & $49(22-59)$ & 0.538 \\
\hline Gender, Female, no. (percent) & $24(41)$ & $13(46)$ & $11(35)$ & 0.393 \\
\hline $\begin{array}{l}\text { Bone marrow blasts at diagnosis } \\
\text { [percent] median (range) }\end{array}$ & $63(20-96)$ & $68.5(20-94)$ & $62.5(20.5-96)$ & 0.792 \\
\hline $\begin{array}{l}\text { WBC count at diagnosis } \\
\text { [Gpt/L] median (range) }\end{array}$ & $26.9(1.3-191.4)$ & $38(1.4-191.4)$ & $18.8(1.3-189.1)$ & 0.370 \\
\hline $\begin{array}{l}\text { Platelet count at diagnosis } \\
\text { [Gpt/L] median (range) }\end{array}$ & $52(13-357)$ & $61(13-231)$ & $50(13-357)$ & 0.970 \\
\hline $\begin{array}{l}\text { Serum LDH at diagnosis } \\
\text { [U/I] median (range) }\end{array}$ & $707(145-4945)$ & $694(186-4945)$ & $768(145-3973)$ & 0.439 \\
\hline FLT3-ITD mutational status, positive, no. (percent) & $12(25)$ & $8(29)$ & $4(13)$ & 0.135 \\
\hline NPM1 mutational status, NPM1 mut, no. (percent) & $21(36)$ & $10(37)$ & $11(35)$ & 0.902 \\
\hline \multicolumn{5}{|l|}{ Combined NPM1/FLT3 status, no. (percent) } \\
\hline NPM1 wt/FLT3 wt & $32(55)$ & $14(52)$ & $18(58)$ & \multirow[t]{4}{*}{0.413} \\
\hline NPM1 wt/FLT3 mut & $5(9)$ & $3(11)$ & $2(6)$ & \\
\hline NPM1 mut/FLT3 wt & $14(24)$ & $5(18)$ & $9(29)$ & \\
\hline NPM1 mut/FLT3 mut & $7(12)$ & $5(18)$ & $2(6)$ & \\
\hline \multicolumn{5}{|l|}{ Disease status at diagnosis, no. (percent) } \\
\hline De novo AML & $53(90)$ & $28(100)$ & $25(81)$ & \multirow[t]{3}{*}{0.049} \\
\hline tAML & $5(8)$ & $0(0)$ & $5(16)$ & \\
\hline mdsAML & $1(2)$ & $0(0)$ & $1(3)$ & \\
\hline \multicolumn{5}{|l|}{ FAB subtypes at diagnosis, no. (percent) } \\
\hline MO & $2(3)$ & $0(0)$ & $2(6)$ & \multirow[t]{11}{*}{0.003} \\
\hline M1 & $11(19)$ & $9(32)$ & $2(6)$ & \\
\hline $\mathrm{M} 2$ & $14(24)$ & $10(36)$ & $4(13)$ & \\
\hline M4 & $9(15)$ & $2(7)$ & $7(23)$ & \\
\hline M4eo & $2(3)$ & $2(7)$ & $0(0)$ & \\
\hline M5a & $11(19)$ & $1(4)$ & $10(32)$ & \\
\hline M5b & $2(3)$ & $0(0)$ & $2(6)$ & \\
\hline M6 & $2(3)$ & $1(4)$ & $1(3)$ & \\
\hline M7 & $1(2)$ & $0(0)$ & $1(3)$ & \\
\hline RAEB-T & $3(5)$ & $1(4)$ & $2(6)$ & \\
\hline M5 other & $2(3)$ & $2(7)$ & $0(0)$ & \\
\hline \multicolumn{5}{|l|}{ Cytogenetic subgroups, no. (percent) } \\
\hline High risk & $19(32)$ & $3(11)$ & $16(52)$ & \multirow[t]{3}{*}{0.001} \\
\hline Standard risk & $33(56)$ & $19(68)$ & $14(45)$ & \\
\hline Low risk & $7(12)$ & $6(21)$ & $1(3)$ & \\
\hline \multicolumn{5}{|l|}{ Detailed karyotypes, no. (percent) } \\
\hline Complex karyotype & $12(20)$ & $3(11)$ & $9(29)$ & 0.081 \\
\hline Normal karyotype & $18(32)$ & $12(44)$ & $6(20)$ & 0.047 \\
\hline $\mathrm{t}(8 ; 21)$ & $4(7)$ & $3(11)$ & $1(3)$ & 0.253 \\
\hline inv16 & $3(5)$ & $3(11)$ & $0(0)$ & 0.061 \\
\hline monosomy 5 & $2(3)$ & $0(0)$ & $2(6)$ & 0.171 \\
\hline
\end{tabular}


Table 3 Characteristics of LOX-low/LOX-high extramedullary AML (Continued)

\begin{tabular}{|c|c|c|c|c|}
\hline & \multicolumn{4}{|l|}{$\begin{array}{l}\text { all EM AML } \\
n=59\end{array}$} \\
\hline & $\begin{array}{l}\text { all EM AML } \\
n=59\end{array}$ & $\begin{array}{l}\text { LOX-low group } \\
n=28,60 \%\end{array}$ & $\begin{array}{l}\text { LOX-high group } \\
n=31,40 \%\end{array}$ & $\overline{p \text {-value }}$ \\
\hline del5q & $3(5)$ & $1(4)$ & $2(6)$ & 0.615 \\
\hline monosomy 7 & $1(2)$ & $1(4)$ & $0(0)$ & 0.289 \\
\hline del7q & $3(5)$ & $1(4)$ & $2(6)$ & 0.615 \\
\hline monosomy other than chromosomes 5 or 7 & $3(5)$ & $1(4)$ & $2(6)$ & 0.615 \\
\hline trisomy 8 & $11(19)$ & $3(11)$ & $8(26)$ & 0.137 \\
\hline$t(9 ; 11)$ & $3(5)$ & $0(0)$ & $3(10)$ & 0.091 \\
\hline abnl(11q23) & $10(17)$ & $1(4)$ & $9(29)$ & 0.009 \\
\hline
\end{tabular}

Table 3 Univariate results of EM-AML patients' characteristics, divided in LOX-low and LOX-high group

Abbreviations: $A M L$ acute myeloid leukemia, FAB French American British classification of acute leukemia, FLT3-ITD FMS-like tyrosine kinase 3 internal tandem duplication, $L D H$ lactate dehydrogenase, MDS myelodysplastic syndrome, NPM1 Nucleophosmin 1, RAEB refractory anemia with excess blasts, $t-A M L$ therapy-related $\mathrm{AML}$, mdsAML AML with preceding MDS, WBC white blood count

suggests a potentially pathophysiological relevant mechanism involved in extramedullary homing and growth of AML and may offer further insights into AML biology. The prognostic heterogeneous group of AML patients with extramedullary disease can be separated in those with superior and those with inferior survival by applying the peripheral blood lithium-heparin plasma LOX expression level at diagnosis. This might be of importance for future applications in clinical as well as in scientific settings since so far, the survival of AML patients with extramedullary disease at diagnosis has been discussed controversially. Importantly, our data need to be confirmed in an independent validation patient cohort which might prove difficult since most large AML trials do not collect and bank plasma samples. Further experimental studies are needed to address the functional modalities of how LOX is regulated and how it contributes to migratory and adhesion properties in AML. Possible caveats for future studies addressing these questions might be that few AML trials include the systematic screening, documentation and analysis of extramedullary AML - a phenomenon that already led to an underreporting of extramedullary AML after allogeneic HSCT [39]. Even more so, data exist demonstrating that allogeneic HSCT performed as second allogeneic transplantation after relapsed disease or in a haploidentical setting might be associated with a higher prevalence of extramedullary AML at relapse, implicating clonal evolution and immune-escape phenomena involved as well as varying susceptibilities for AML seeds in different tissues [40]. In clinical reality, only those extramedullary AML manifestations which are clinically apparent or identified by imaging studies [41, 42] and which are histologically confirmed are taken into account in scientific analyses like ours $[11,13,40-42]$. This could theoretically translate into an underreporting of extramedullary AML in general. One approach that has been proposed in this context is the detection of WT1 expression by quantitative PCR which was reported to predict extramedullary AML. Patients with extramedullary relapse had high expression levels in the peripheral blood whereas the WT1 level in the bone marrow remained negative [43]. Furthermore, AML1-MTG8 expression levels were shown to possibly predict EM relapse, specifically in t $(8 ; 21)$ AML [44].

Further indicators linking LOX to extramedullary manifestation of AML in our report is certain characteristics known to be more common in extramedullary AML were more frequently observed in the LOX-high group, i.e. higher numbers of patients with monocytic morphology and certain karyotypic abnormalities that have been associated with extramedullary AML such as e.g. complex aberrant karyotypes, trisomy of chromosome 8 , or $11 \mathrm{q} 23$ abnormalities amongst others [8, 17, 19, 20, 45, 46]. Worth considering for future studies and analyses on the pathophysiology and the genetic evolution of extramedullary AML is the so far unacquainted matter that karyotypic features of AML clones residing in the bone marrow might be different from those residing in extramedullary sites as demonstrated by array-CGH technology [18].

However, without the existence of a homogenous tumor population within one individual it was speculated by Fidler et al. that in epithelial cancers rare subpopulations of malignant clones exist which are endowed with some or several "metastasis-promoting functions" [47]. Furthermore, it was hypothesized that highly metastatic clones from a heterogenous tumor cell population have a higher rate of genetic variation and a higher rate of mutations than non-metastatic clones from the same tumor cell population [48]. This concurs with the observation of a higher incidence of clonal heterogeneity in extramedullary AML (i.e. complex karyotypes) [18]. Alas, studies performing comparative genetic analyses in extramedullary AML samples and bone marrow or blood samples specifically are needed in order to 


\section{A. Overall Survival of LOX-high and LOX-low in extramedullary AML patients.}

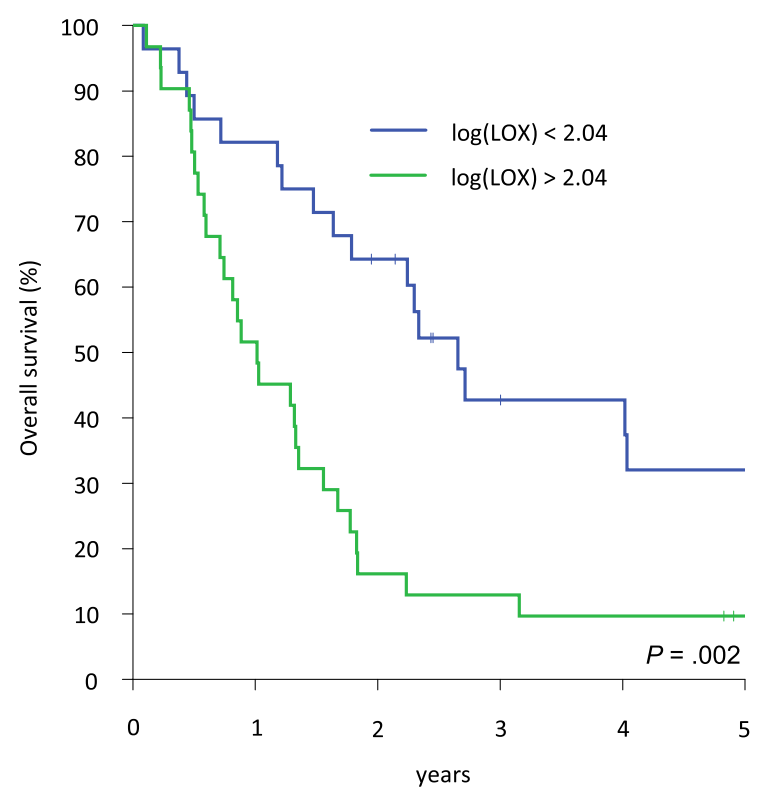

B. Event-free Survival of LOX-high and LOX-low in extramedullary AML

patients.

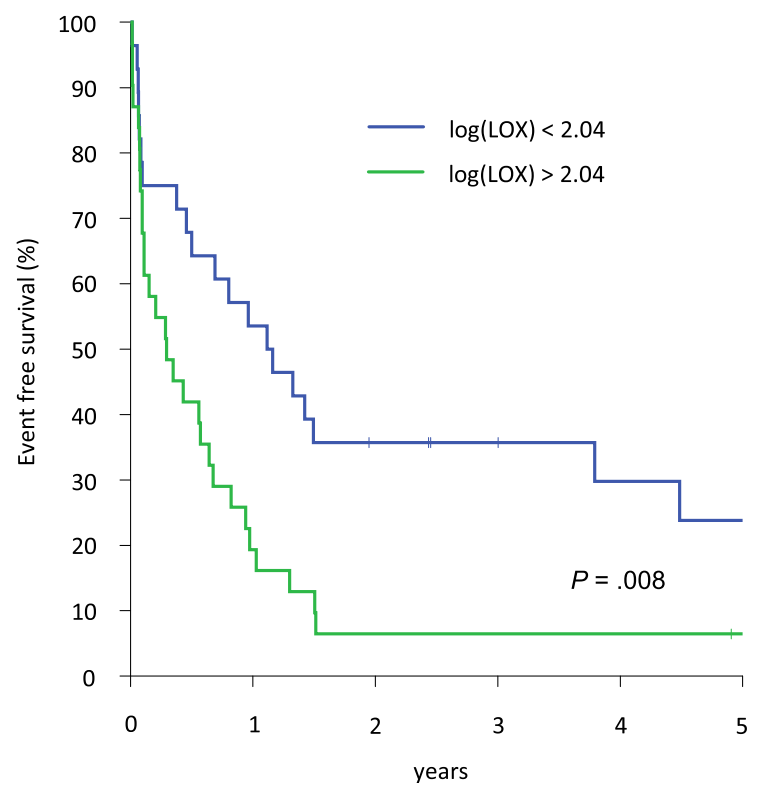

Fig. 2 a and $\mathbf{b}$ Overall Survival (OS) (Fig. 1a) and Event-free Survival (EFS) of 59 AML patients with extramedullary disease dichotomized to the LOX-high $(n=31)$ and LOX-low $(n=28)$ group. LOX-high patients are denoted in green, LOX-low patients are denoted in blue color. The logarithmic logLOX value $=2.0403(109 \mathrm{ng} / \mathrm{mL})$ was used to dichotomize all patients in both groups, respectively. For LOX-high patients 3-year OS was 13\% (95\% Cl, 1-25\%) and for LOX-low patients 43\% (95\% Cl, 23-63\%), $p=.002$. Three-year EFS for LOX-high patients was 6\% (95\% Cl, 0-15\%) and for LOX-low patients 36\% (95\% Cl, 17-54\%), $p=0.008$

exploit the different functional implications of different AML clones. One aspect of the leukemia cell invadosome in extramedullary infiltration has been studied revealing that interactions between matrix-metalloproteinase- 9 and leukocyte $\beta 2$-Integrin are necessary for migration, for progression, and chemosensitivity in AML [49, 50].

While the three-dimensional structure of the human LOX protein has not been resolved so far, it furthermore 
needs to be explored which isoforms of LOX are relevant in the context of AML and whether its regulation through HIFs in hypoxia, its ability to recruit CD11b myeloid cells which adhere to crosslinked collagen and produce matrix-metalloproteinase- 2 enhancing premetastatic niche formation is of relevance in AML. Also the ability of LOX to increase vascular endothelial growth factor (VEGF) expression and secretion which promotes angiogenesis needs to be reflected in this context [51]. Finally, follow-up plasma samples during treatment and determination of LOX activity could reflect a response to treatment through decrease in LOX activity as one might hypothesize in AML and in patients with extramedullary AML, specifically.

\section{Conclusion}

The correlation between high LOX expression and extramedullary AML suggests a potentially relevant mechanism involved in extramedullary adhesion, migration, and growth of AML and may offer further insights into AML homing biology and therapeutic targeting options. However, one must speculate that due to the lack of routine screening for extramedullary AML at diagnosis, there might be a considerable amount of unknown extramedullary AML at diagnosis, especially in cases without clinical apparent extramedullary manifestations, for which LOX could also be a potential marker for prognosis. A subsequent approach in order to modify or even selectively inhibit LOX might be reasonable for an attempt to improve the clinically inferior and still dissatisfying outcome of these patients.

\begin{abstract}
Abbreviations
3'UTR: 3' untranslated region; AML: Acute myeloid leukemia; BMP-1: Bone morphogenic protein-1; CR: Complete remission; EFS: Event-free survival; FAB: French-American-British; FISH: Fluorescent in situ hybridization; FLT3ITD: FMS-like tyrosine kinase 3 internal tandem duplication; HIF-1: Hypoxiainducible factor-1; HSCT: Hematopoietic stem cell transplantation; IRB: Institutional review board; LDH: Lactate dehydrogenase; LOX: Lysyl oxidase; MAC/MAMAC/MAC: Mitoxantrone, amsacrine, high-dose cytarabine; mdsAML: AML with preceding MDS; MDS-EB2: Myelodysplastic syndrome with excess blasts > 9\%; miR-10a: microRNA 10a; NPM1: Nucleophosmin 1; OS: Overall Survival; SAL: Study Alliance Leukemia; t-AML: therapy-related AML; VEGF: vascular endothelial growth factor; WBC: white blood count; WHO: World Health Organization; WT1: Wilms' tumor protein 1
\end{abstract}

\section{Acknowledgements}

The participation of nurses, staff and doctors of centers of the Study Alliance Leukemia (SAL) is highly acknowledged.

\section{Authors' contributions}

DK and FS performed the research. FS designed the research study. FS, DK and $C D$ performed the experiments. All authors analyzed and interpreted the data. DK and FS wrote the paper. All authors commented on previous versions of the manuscript. All authors read and approved the final manuscript.

\section{Funding}

This work was partially sponsored by Grants from 'Deutsche Forschungsgemeinschaft' (DFG) SFB 655, B7 to FS and MB, and from the 'Cancer Research Foundation' to FS. FS received research funding from the
University of Dresden (MeDDrive Grant). Open Access Funding by the Publication Fund of the TU Dresden.

\section{Availability of data and materials}

The datasets generated and analyzed during the study are available from the corresponding author on reasonable request.

\section{Ethics approval and consent to participate}

All procedures followed were in accordance with the ethical standards of the responsible committee on human experimentation (institutional and national) and with the Helsinki Declaration of 1975, as revised in 2008. The study had been approved by the institutional review board (IRB) of the University of Dresden and all IRBs of participating centers of the SAL. Informed consent was obtained from all patients for being included in the study.

\section{Consent for publication}

All authors approved the manuscript and gave their consent for publication.

\section{Competing interests}

The authors declare that they have no competing interests.

\section{Author details}

${ }^{1}$ Department of Internal Medicine I, University Hospital Carl Gustav Carus, Technical University of Dresden, Fetscherstrasse 74, 01307 Dresden, Germany. ${ }^{2}$ Department of hematology, oncology and palliative care,

Rems-Murr-Klinikum, Winnenden, Germany.

Received: 2 April 2020 Accepted: 5 June 2020

Published online: 12 June 2020

\section{References}

1. Carmeliet P, Jain RK. Molecular mechanisms and clinical applications of angiogenesis. Nature. 2011;473(7347):298-307.

2. Gupta GP, Massagué J. Cancer metastasis: building a framework. Cell. 2006; 127(4):679-95.

3. Höckel M, Vaupel P. Tumor hypoxia: definitions and current clinical, biologic and molecular aspects. J Natl Cancer Inst. 2001;93(4):266-76.

4. Kaplan RN, et al. VEGFR1-positive haematopoietic bone marrow progenitors initiate the pre-metastatic niche. Nature. 2005:438(7069):820-7.

5. Erler JT, et al. Hypoxia-induced lysyl oxidase is a critical mediator of bone marrow cell recruitment to form the premetastatic niche. Cancer Cell. 2009; 15(1):35-44.

6. Ohanian $\mathrm{M}$, et al. Is acute myeloid leukemia a liquid tumor? Int J Cancer. 2013;133(3):534-43.

7. Bakst RL, et al. How I treat extramedullary acute myeloid leukemia. Blood. 2011:118(14):3785-93.

8. Lee $\mathrm{KH}$, et al. Bone marrow vs extramedullary relapse of acute leukemia after allogeneic hematopoietic cell transplantation: risk factors and clinical course. Bone Marrow Transplant. 2003;32(8):835-42.

9. Simpson DR, et al. High incidence of extramedullary relapse of AML after busulfan/cyclophosphamide conditioning and allogeneic stem cell transplantation. Bone Marrow Transplant. 1998;22(3):259-64.

10. Solh $\mathrm{M}$, et al. Extramedullary relapse of acute myelogenous leukemia after allogeneic hematopoietic stem cell transplantation: better prognosis than systemic relapse. Biol Blood Marrow Transplant. 2012;18(1):106-12.

11. Choi SJ, et al. Treatment of relapsed acute myeloid leukemia after allogeneic bone marrow transplantation with chemotherapy followed by GCSF-primed donor leukocyte infusion: a high incidence of isolated extramedullary relapse. Leukemia. 2004;18(11):1789-97.

12. Yoshihara S, et al. Incidence of extramedullary relapse after haploidentical $\mathrm{SCT}$ for advanced AML/myelodysplastic syndrome. Bone Marrow Transplant. 2012;47(5):669-76.

13. Stölzel $F$, et al. The prevalence of extramedullary acute myeloid leukemia detected by 18FDG-PET/CT: final results from the Prospective PETAML Trial. Haematologica. 2019. https://doi.org/10.3324/haematol.2019.223032.

14. Byrd JC, et al. Extramedullary leukemia adversely affects hematologic complete remission rate and overall survival in patients with $\mathrm{t}(8 ; 21)$ (q22; q22): results from Cancer and leukemia group B 8461. J Clin Oncol. 1997; 15(2):466-75. 
15. Chang $\mathrm{H}$, et al. Molecular cytogenetic abnormalities in patients with concurrent chronic lymphocytic leukemia and multiple myeloma shown by interphase fluorescence in situ hybridization: evidence of distinct clonal origin. Cancer Genet Cytogenet. 2004;148(1):44-8.

16. Tallman MS, et al. Acute monocytic leukemia (French-American-British classification M5) does not have a worse prognosis than other subtypes of acute myeloid leukemia: a report from the eastern cooperative oncology group. J Clin Oncol. 2004;22(7):1276-86.

17. Deeb $\mathrm{G}$, et al. Genomic profiling of myeloid sarcoma by array comparative genomic hybridization. Genes Chromosom Cancer. 2005;44(4):373-83.

18. Pileri SA, et al. Myeloid sarcoma: clinico-pathologic, phenotypic and cytogenetic analysis of 92 adult patients. Leukemia. 2007;21(2):340-50

19. Tsimberidou AM, et al. Outcome in patients with nonleukemic granulocytic sarcoma treated with chemotherapy with or without radiotherapy. Leukemia. 2003;17(6):1100-3.

20. Döhner K, et al. Mutant nucleophosmin (NPM1) predicts favorable prognosis in younger adults with acute myeloid leukemia and normal cytogenetics: interaction with other gene mutations. Blood. 2005;106(12):3740-6.

21. Falini B, et al. Cytoplasmic mutated nucleophosmin (NPM) defines the molecular status of a significant fraction of myeloid sarcomas. Leukemia. 2007;21(7):1566-70.

22. Garzon R, et al. Distinctive microRNA signature of acute myeloid leukemia bearing cytoplasmic mutated nucleophosmin. Proc Natl Acad Sci U S A. 2008;105(10):3945-50.

23. Ovcharenko D, et al. miR-10a overexpression is associated with NPM1 mutations and MDM4 downregulation in intermediate-risk acute myeloid leukemia. Exp Hematol. 2011;39(10):1030-42 e7.

24. Papadantonakis $\mathrm{N}$, et al. Megakaryocyte pathology and bone marrow fibrosis: the lysyl oxidase connection. Blood. 2012;120(9):1774-81.

25. Tadmor $\mathrm{T}$, et al. The expression of lysyl-oxidase gene family members in myeloproliferative neoplasms. Am J Hematol. 2013;88(5):355-8.

26. Dawes CS, et al. Enzyme-immobilized hydrogels to create hypoxia for in vitro cancer cell culture. J Biotechnol. 2017;248:25-34.

27. Denko NC, et al. Investigating hypoxic tumor physiology through gene expression patterns. Oncogene. 2003;22(37):5907-14.

28. Pez F, et al. The HIF-1-inducible lysyl oxidase activates HIF-1 via the Akt pathway in a positive regulation loop and synergizes with HIF-1 in promoting tumor cell growth. Cancer Res. 2011;71(5):1647-57.

29. Cox TR, et al. LOX-mediated collagen crosslinking is responsible for fibrosisenhanced metastasis. Cancer Res. 2013;73(6):1721-32.

30. Wang $Y$, et al. Gene-expression profiles to predict distant metastasis of lymph-node-negative primary breast cancer. Lancet. 2005;365(9460):671-9.

31. Schaich M, et al. High-dose Cytarabine consolidation with or without additional Amsacrine and Mitoxantrone in acute myeloid leukemia: results of the prospective randomized AML2003 trial. J Clin Oncol. 2013;31(17): 2094-102.

32. Shaffer $L G$ et al. An international system for human cytogenetic nomenclature (ISCN). 2009.

33. Thiede $C$, et al. Prevalence and prognostic impact of NPM1 mutations in 1485 adult patients with acute myeloid leukemia (AML). Blood. 2006;107(10): 4011-20.

34. Thiede $\mathrm{C}$, et al. Analysis of FLT3-activating mutations in 979 patients with acute myelogenous leukemia: association with FAB subtypes and identification of subgroups with poor prognosis. Blood. 2002;99(12):4326-35.

35. Swerdlow SH, et al. WHO Classification of Tumours of Haematopoietic and Lymphoid Tissues; 2008. p. 140-1.

36. Palamakumbura AH, Trackman PC. A fluorometric assay for detection of lysyl oxidase enzyme activity in biological samples. Anal Biochem. 2002; 300(2):245-51.

37. Cheson $\mathrm{BD}$, et al. Revised recommendations of the international working Group for Diagnosis, standardization of response criteria, treatment outcomes, and reporting standards for therapeutic trials in acute myeloid leukemia. J Clin Oncol. 2003;21(24):4642-9.

38. Contal C, O'Quigley J. An application of changepoint methods in studying the effect of age on survival in breast cancer. Comput Stat Data Anal. 1999; 30:253-70.

39. Bekassy AN, et al. Granulocytic sarcoma after allogeneic bone marrow transplantation: a retrospective European multicenter survey. Acute and chronic leukemia working parties of the European Group for Blood and Marrow Transplantation. Bone Marrow Transplant. 1996;17(5):801-8.
40. Yoshihara S, et al. Extramedullary relapse of acute myeloid leukemia after allogeneic hematopoietic stem cell transplantation: an easily overlooked but significant pattern of relapse. Biol Blood Marrow Transplant. 2012;18(12):1800-7.

41. Buck AK, et al. First demonstration of leukemia imaging with the proliferation marker 18F-fluorodeoxythymidine. J Nucl Med. 2008;49(11): 1756-62.

42. Stölzel F, et al. ${ }^{18} \mathrm{~F}$-FDG-PET/CT for detection of extramedullary acute myeloid leukemia. Haematologica. 2011;96(10):1552-6.

43. Kwon $\mathrm{M}$, et al. Evaluation of minimal residual disease by real-time quantitative PCR of Wilms' tumor 1 expression in patients with acute myelogenous leukemia after allogeneic stem cell transplantation: correlation with flow cytometry and chimerism. Biol Blood Marrow Transplant. 2012; 18(8):1235-42.

44. Tamaki H, et al. Molecular detection of AML1-MTG8-positive cells in peripheral blood from a patient with isolated extramedullary relapse of $\mathrm{t}$ (8; 21) acute myeloid leukemia. Leukemia. 2009;23(2):424-6.

45. Tsimberidou AM, et al. Myeloid sarcoma is associated with superior eventfree survival and overall survival compared with acute myeloid leukemia. Cancer. 2008;113(6):1370-8.

46. Schaich $M$, et al. Prognosis of acute myeloid leukemia patients up to 60 years of age exhibiting trisomy 8 within a non-complex karyotype: individual patient data-based meta-analysis of the German acute myeloid leukemia intergroup. Haematologica. 2007;92(6):763-70.

47. Fidler IJ. The pathogenesis of cancer metastasis: the 'seed and soil' hypothesis revisited. Nat Rev Cancer. 2003;3(6):453-8.

48. Cifone MA, Fidler IJ. Increasing metastatic potential is associated with increasing genetic instability of clones isolated from murine neoplasms. Proc Natl Acad Sci U S A. 1981;78(11):6949-52.

49. De Toni F, et al. A crosstalk between the Wnt and the adhesion-dependent signaling pathways governs the chemosensitivity of acute myeloid leukemia. Oncogene. 2006;25(22):3113-22

50. Stefanidakis $M$, et al. Role of leukemia cell invadosome in extramedullary infiltration. Blood. 2009:114(14):3008-17.

51. Baker AM, et al. Lysyl oxidase plays a critical role in endothelial cell stimulation to drive tumor angiogenesis. Cancer Res. 2013;73(2):583-94.

\section{Publisher's Note}

Springer Nature remains neutral with regard to jurisdictional claims in published maps and institutional affiliations.

Ready to submit your research? Choose BMC and benefit from:

- fast, convenient online submission

- thorough peer review by experienced researchers in your field

- rapid publication on acceptance

- support for research data, including large and complex data types

- gold Open Access which fosters wider collaboration and increased citations

- maximum visibility for your research: over $100 \mathrm{M}$ website views per year

At $\mathrm{BMC}$, research is always in progress.

Learn more biomedcentral.com/submissions 